\title{
Evaporation of grain-surface species by shock waves onto a forming protoplanetary disk
}

\author{
Yuri Aikawa ${ }^{1}$, Takuhiro Aota $^{2}$ and Tsuyoshi Inoue ${ }^{3}$ \\ ${ }^{1}$ Center for Computer Sciences, University of Tsukuba \\ email: aikawa@ccs.tsukuba.ac.jp \\ ${ }^{2}$ Fuji Soft \\ ${ }^{3}$ Division of Theoretical Astronomy, National Astronomical Observatory of Japan
}

\begin{abstract}
We investigate the sputtering and thermal desorption of various grain-surface species in one dimensional steady-state shock models motivated by the recent detection of SO emission towards class 0-I protostars. We find that the thermal desorption is more efficient at higher densities, while the efficiency of sputtering is independent of density. SO is completely desorbed, if the accretion velocity is higher than $\sim 2 \mathrm{~km} \mathrm{~s}^{-1}$ and $\sim 4 \mathrm{~km} \mathrm{~s}^{-1}$, with the pre-shock density of $10^{9} \mathrm{~cm}^{-3}$ and $10^{8} \mathrm{~cm}^{-3}$, respectively. The column density of warm post-shock gas is found to be $N \sim 10^{21} \mathrm{~cm}^{-2}$. If the abundance of SO ice is $\sim 10^{-7}$ relative to hydrogen in the pre-shock material, SO emission around L1527 can be explained by the sublimation at the accretion shock.
\end{abstract}

Keywords. astrochemistry, protoplanetary disk

\section{Introduction}

Atacama Large Millimeter/submillimeter Array observations of class 0-I protostellars detected warm SO emission (Sakai et al.2014a, Sakai et al.2014b, Yen et al.2014). The position-velocity diagrams indicate that SO abundance is enhanced in a ring region of radius $\sim 100 \mathrm{AU}$ and $\sim 300 \mathrm{AU}$ around L1527 and L1489, respectively. Emission lines of carbon chains, on the other hand, show infall motion with rotation around L1527. Considering these velocity profiles, Sakai et al.(2014a) proposed that SO emission arises from the accretion shock at the centrifugal barrier. In the chemical models of protostellar cores (Aikawa et al.2012), SO ice is indeed a major carrier of sulfur. In order to investigate if $\mathrm{SO}$ can be desorbed by a rather weak accretion shock at the radius of $\sim 100 \mathrm{AU}$ (i.e. the pre-shock velocity of $\sim 2 \mathrm{~km} \mathrm{~s}^{-1}$ ), we calculate the desorption of grain surface species in the $1 \mathrm{D}$ steady-state shock model.

\section{Model}

We first calculate the structure (density and temperature of gas) of 1D steady-state shock. Then the motion of dust grains in the flow is calculated referring to Hood \& Horanyi (1991). Dust temperature is determined by the balance between heating (gas-grain energy transfer) and cooling (thermal emission). We consider thermal desorption and sputtering (Draine \& Salpeter 1979, see also Jiménez-Serra et al.2008). The desorption energies of molecules are adopted from Garrod \& Herbst (2006); the value for SO is 2600 $\mathrm{K}$, which is similar to that of $\mathrm{CO}_{2}$. More detailed explanations of the model can be found in Aota et al.(2015). 

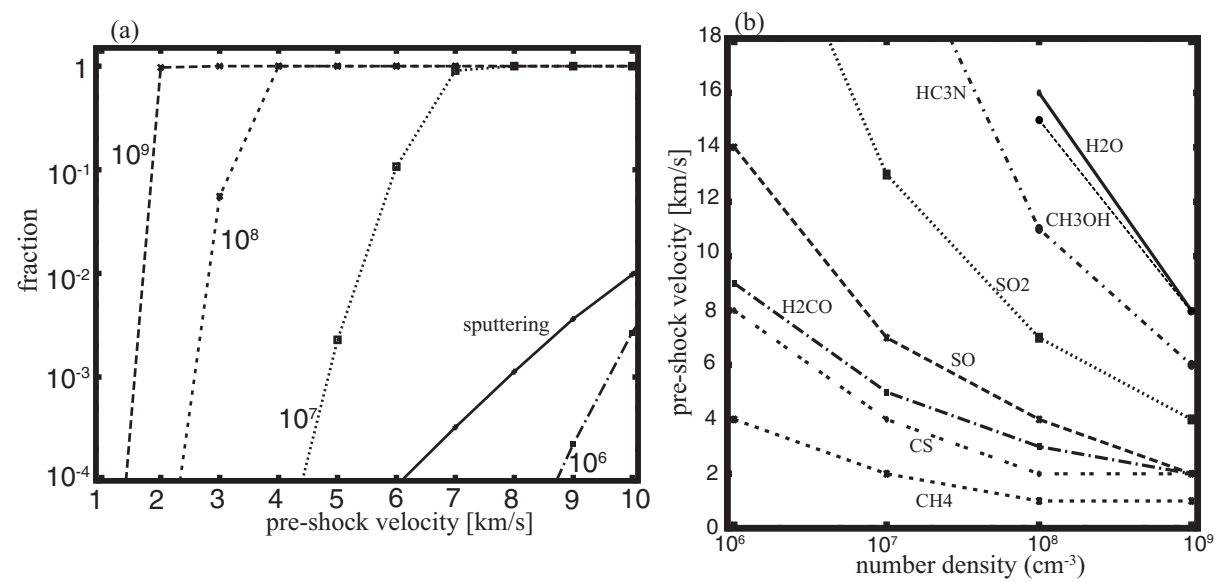

Figure 1. (a) The fraction of thermally desorbed and sputtered SO as a function of pre-shock velocity with the pre-shock gas density $n_{\mathrm{H}}=10^{6}, 10^{7}, 10^{8}$, and $10^{9} \mathrm{~cm}^{-3}$. (b) The pre-shock critical velocity above which various molecules are completely desorbed as a function of the pre-shock density.

\section{Results}

Figure 1 (a) shows the fraction of thermally desorbed and sputtered SO as a function of pre-shock velocity, when the pre-shock gas density is $n_{\mathrm{H}}=10^{6}, 10^{7}, 10^{8}$, and $10^{9}$ $\mathrm{cm}^{-3}$. While the thermal desorption is more efficient in higher densities, the efficiency of sputtering is independent of gas densities. Figure 1 (b) shows the pre-shock critical velocity above which various molecules are completely desorbed as a function of the preshock density. In the observation of L1527, the pre-shock velocity is estimated to be $\sim 2$ $\mathrm{km} \mathrm{s}^{-1}$. If the gas density is $\sim 10^{9} \mathrm{~cm}^{-3}$, SO ice can be completely desorbed by the accretion shock.

The column density of warm $(\sim 100 \mathrm{~K})$ gas in the post-shock region is determined by the cooling timescale of dust grains, and is found to be $\sim 10^{21} \mathrm{~cm}^{-2}$, when the grain size is $0.1 \mu \mathrm{m}$. If the SO ice abundance in the pre-shock material is $10^{-7}$, which is plausible according to the chemical model of protostellar cores (Aikawa et al.2012), the warm SO column density in the post-shock gas reaches $10^{14} \mathrm{~cm}^{-2}$. It is comparable to the estimated column density of SO gas from the observation towards L1527 (Sakai et al.2014a).

\section{References}

Aikawa, Y., Wakalem, V., Hersant, F., Garrod, R. T. \& Herbst, E. 2012 ApJ, 760, 40

Aota, T., Inoue, T. \& Aikawa, Y. 2015, ApJ, 799, 141

Draine, B. T. \& Salpeter, E. E. 1979, ApJ, 231, 77

Garrod, R. T. \& Herbst, E. 2006, A\& $A, 457,927$

Hood, L. L. \& Horanyi, M. 1991, Icarus, 93, 259

Jiménez-Sarra, I., Caselli, P., Martín-Pintado, J. \& Hartquist, T. W. 2008, A $\& A$, 482, 549

Sakai, N. et al. 2014a, Nature, 507, 78

Sakai, N. et al. 2014b, ApJ, 791, 38

Yen et al. 2014, ApJ 793, 1 